\title{
Poly Meta-Aminophenol: Chemical Synthesis, Characterization and Ac Impedance Study
}

\author{
Thenmozhi Gopalasamy, ${ }^{1}$ Mohanraj Gopalswamy, ${ }^{2}$ \\ Madhusudhana Gopichand, ${ }^{1}$ and Jayasanthi Raj ${ }^{1}$ \\ ${ }^{1}$ PG \& Research Department of Chemistry, Auxilium College, Vellore, Tamil Nadu 632006, India \\ ${ }^{2}$ Department of Physics, Martin Luther University of Halle-Wittenberg, 06120 Halle, Germany
}

Correspondence should be addressed to Jayasanthi Raj; shanthijaya02@gmail.com

Received 14 November 2013; Accepted 28 November 2013; Published 19 January 2014

Academic Editor: Yves Grohens

Copyright (C) 2014 Thenmozhi Gopalasamy et al. This is an open access article distributed under the Creative Commons Attribution License, which permits unrestricted use, distribution, and reproduction in any medium, provided the original work is properly cited.

The present work is an investigation of AC impedance behaviour of poly(meta-aminophenol). The polymer was prepared by oxidative chemical polymerization of meta-aminophenol in aqueous $\mathrm{HCl}$ using ammonium persulfate as an oxidant at $0-3^{\circ} \mathrm{C}$. The synthesized polymer was characterized by GPC, Elemental analysis, UV-VIS-NIR, FT-IR, ${ }^{1} \mathrm{H}$ NMR, XRD, SEM, and TGADTA. The AC conductivity and dielectric response were measured at a temperature range from 303 to $383 \mathrm{~K}$ in the frequency range of $20 \mathrm{~Hz}$ to $10^{6} \mathrm{~Hz}$. The AC conductivity data could be described by the relation $\sigma_{\mathrm{ac}} \omega=A \omega^{S}$, where the parameter "S" and $R_{b}$ values decrease in the entire range of study and hence follow Correlated Barrier Hopping conduction mechanism. Both dielectric constant and dielectric loss increase with the decrease of frequency exhibiting strong interfacial polarization at low frequency and the dissipation factor also decreases with frequency. Complex electric modulus and dissipation factor exhibit two relaxation peaks, indicating two-phase structure as indicated by a bimodal distribution of relaxation process. The activation energies corresponding to these two relaxation processes were found to be 0.07 and $0.1 \mathrm{eV}$.

\section{Introduction}

Conducting polymers such as polyaniline, polythiophene, and polypyrrole are the subject of scientific interest due to their unique high electrical conductivity and numerous potential applications including rechargeable batteries, antistatic coatings, electromagnetic screens, anticorrosive materials and sensors. Among the conducting polymers, polyaniline attracts greater attention because of its chemical stability under ambient conditions, high electrical conductivity, and simple synthesis [1]. A derivative of polyaniline, aminophenols are interesting electrochemical materials since, unlike aniline and other substituted anilines, they have two groups $\left(-\mathrm{NH}_{2}\right.$ and $\left.-\mathrm{OH}\right)$ which can be oxidized. Therefore, they can show electrochemical behavior resembling anilines and phenols. In polymerization of aminophenol, the relative position of amino and hydroxyl group is important [2]. In recent years, electrical, optical, and dielectric properties of conducting polymers like polyaniline and substituted polyaniline synthesized by chemical oxidation polymerization have been studied in great detail.

Impedance spectroscopy is a very useful technique in solid state electronic system, because it can resolve the conduction components by differentiating between the transports properties of complex systems [3]. The behavior of AC conductivity according to the frequency and the temperature generally follows a similar behavior in all disordered solids. This AC conductivity is often controlled by deep electronic states which one will find in the vicinity of the level of Fermi. At first order, the alternating electric field applied to the material will induce transition of charges on deep or shallow defect centres. Following the frequency, these charges could migrate at long range or short range distance in the material. The temperature will influence this AC conductivity 
Step I

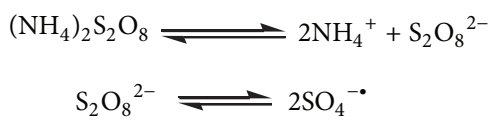<smiles>[R20]C1CC1C1C=CC(=[NH2+])C=C1O</smiles><smiles>[NH3+]c1ccc(Nc2ccc(N=C3C=CC([NH2+]c4cccc(O)c4)C(O)=C3)c(O)c2)c(O)c1</smiles>

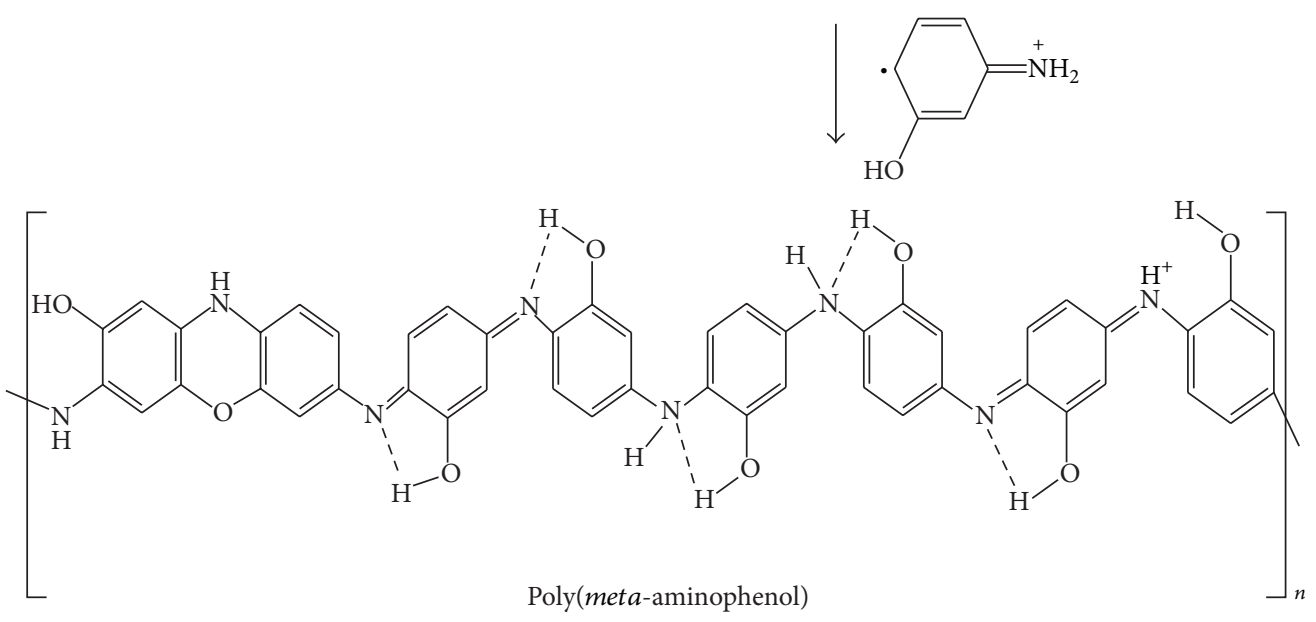

SCHeme 1: Proposed mechanism of polymerization of meta-aminophenol.

particularly at long range distance where charges have time to be subject to the influence of the temperature $[4,5]$.

In the present work, poly(meta-aminophenol) (PMAP) was synthesized by chemical oxidation method and characterized by different spectroscopic techniques. An attempt has been made to investigate the AC conductivity and dielectric behavior of PMAP. Interesting results are observed from frequency- and temperature-dependent AC conductivity and dielectric response of the synthesized polymer at $0-3^{\circ} \mathrm{C}$.

\section{Experimental Method}

2.1. Synthesis of Poly(meta-aminophenol). $0.1 \mathrm{M}$ monomer and $1 \mathrm{M} \mathrm{HCl}$ were taken in a beaker containing $50 \mathrm{~mL}$ toluene. Polymerization reaction was started by dropwise addition of aqueous solution of APS $\{0.1 \mathrm{M}$ APS in $100 \mathrm{~mL}$ distilled water $\}$ and the reaction was carried out for $12 \mathrm{~h}$ at $0-3^{\circ} \mathrm{C}$ with constant stirring. Polymerization was stopped by addition of $50 \mathrm{~mL}$ methanol. The PMAP precipitated was filtrated and washed with excess amount of water, methanol, and acetone to remove excess of $\mathrm{HCl}$ and APS. The powder of PMAP was then dried at $50^{\circ} \mathrm{C}$ for $24 \mathrm{~h}$. Based on our observation as well as spectral and elemental analyses of the synthesized polymer we have proposed a polymerization reaction as shown in Scheme 1 .

2.2. Characterization. Molecular weight of PMAP was determined by gel permeation chromatography using Styragel columns and a refractive detector (Waters, model R 4000) with THF as the mobile phase. Carbon-hydrogen-nitrogen analysis of PMAP was carried out by a microanalytical technique using an Elementar Vario EL 3 elemental analyzer. UVVIS-NIR spectra of PMAP dissolved in DMSO solvent and were obtained using Varian, Cary-5000 spectrophotometer in the range of 200-2500 $\mathrm{nm}$. The FT-IR spectrum of PMAP was recorded by Thermo Nicolet, Avatar 370 Spectrophotometer. The spectrum of the dry polymer powder in $\mathrm{KBr}$ pellet was recorded from $500 \mathrm{~cm}^{-1}$ to $4000 \mathrm{~cm}^{-1}$. ${ }^{1} \mathrm{H} \mathrm{NMR}$ spectra of PMAP were recorded in a Bruker $(600 \mathrm{MHz})$ instrument using $\mathrm{d}_{6}$-DMSO as solvent. X-ray diffraction (XRD) scan was done with Bruker AXS D8 Advance diffractometer at room temperature using $\mathrm{Cu} \mathrm{K \alpha}(\lambda=1.5406 \AA)$. The $2 \theta$ angle range was from $0^{\circ}$ to $70^{\circ}$. Scanning Electron Microscopy (SEM) was obtained by JEOL JSM 6390LV for PMAP. 
Thermogravimetric analysis (TGA-DTA) was carried out in nitrogen atmosphere at a heating rate $10^{\circ} \mathrm{C} / \mathrm{min}$ up to $750^{\circ} \mathrm{C}$ temperature by Perkin Elmer, Diamond TG/DTA analyzer.

The electrical characterization measurements were made in the temperature range 303 to $383 \mathrm{~K}$ by the complex impedance method. The impedance measurements were carried out on the powder samples, which were made into circular pellets of $8 \mathrm{~mm}$ diameter at a pelletizing pressure of 5 ton $\mathrm{cm}^{-2}$, using a Hewlett Packard model HP 4284A Precision LCR Meter in the frequency range $20 \mathrm{~Hz}$ to $10^{6} \mathrm{~Hz}$ by placing the sample pellets in between two silver electrodes. Complex impedance and modulus spectral formalisms were employed for the analysis of these data. The electrical conductivity $\left(\sigma_{\mathrm{ac}}\right)$ of the polymer was estimated using the relationship

$$
\sigma_{\mathrm{ac}}=\frac{t}{R_{b} A},
$$

where $t$ is the thickness of the sample, $A$ is the area of cross section, and $R_{b}$ is the bulk resistance of the sample.

\section{Results and Discussion}

3.1. Molecular Weight and Elemental Analysis. The molecular weight of the soluble portion of the PMAP was determined by gel permeation chromatography performed in THF. The number-average molecular weight $\left(M_{n}\right)$, weight-average molecular weight $\left(M_{w}\right)$, and poly dispersity index $\left(M_{w} / M_{n}\right)$ values were found to be 56663,60455 , and 1.06 , respectively. The chromatogram was unimodal and has no traces of monomers, oligomers or high molecular masses. The polymerization process provided polymer with poly dispersity index is 1.06 , this value demonstrated that no cross-linking or other by-products originating from parasitic reactions were present [6].

The elemental analysis data of PMAP contains carbon $53 \%$, hydrogen $4.3 \%$, nitrogen $8.2 \%$, and sulfur $0.7 \%$. Elemental analysis result indicates some sulfur incorporates in PMAP due to the salt formation of liberated sulfuric acid from the reduction of oxidant APS with $-\mathrm{N}$ - present within the polymer chain [7].

3.2. UV-VIS-NIR Analysis. The UV-VIS-NIR absorption spectrum of PMAP was recorded in DMSO as shown in Figure 1. The absorption spectrum shows two distinct absorption peaks at around 289 and $585 \mathrm{~nm}$. The first absorption peak at $289-310 \mathrm{~nm}(4.29-4.0 \mathrm{eV})$ is assigned to the $\pi-\pi^{*}$ transition which is related to the extent of conjugation length. The second absorption peak at $571-610 \mathrm{~nm}$ is assigned to $n-\pi^{*}$ transition between the HOMO orbital of the benzenoid ring and the LUMO orbital of the quinoid ring. It is sensitive to the overall oxidation state of the polymer [8]. In addition, the band at $850 \mathrm{~nm}(1.45 \mathrm{eV})$ has been assigned to $-\mathrm{NH}_{+}{ }^{2}$ species which is generated on doping and a correlation between the intensity of this peak and the conductivity of the sample seems to exist [9].

3.3. FT-IR Analysis. The representative FT-IR spectrum of PMAP is shown in Figure 2. A broad peak that appears in

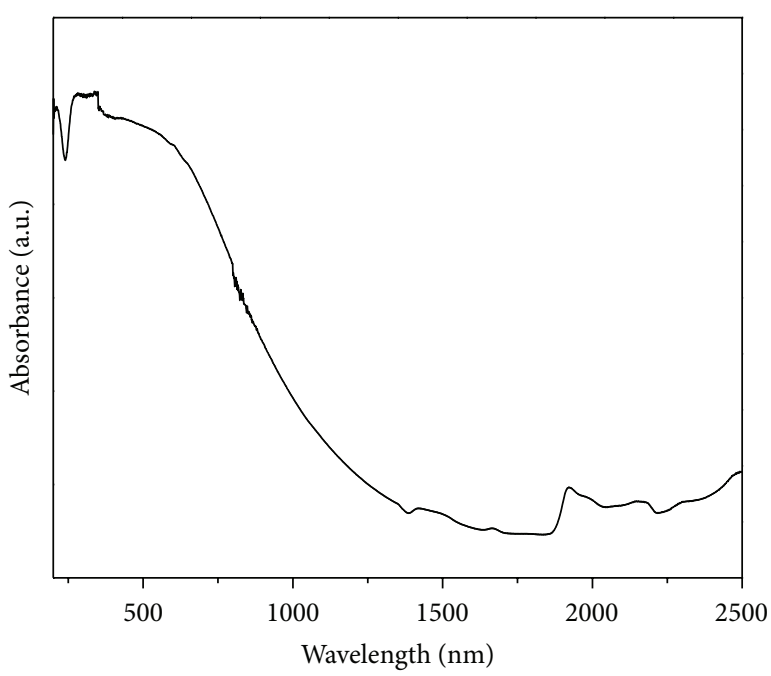

FIGURE 1: UV-VIS-NIR spectra of PMAP in DMSO solvent.

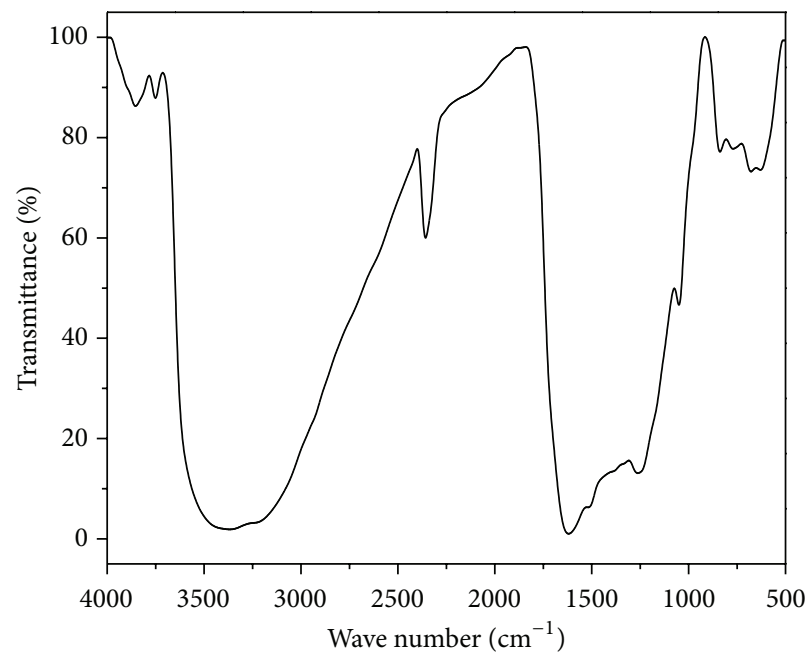

FIGURE 2: FT-IR spectra of PMAP.

the region $3700-1850 \mathrm{~cm}^{-1}$ is due to the stretching of aromatic $\mathrm{C}-\mathrm{H}$, hydrogen bonded $-\mathrm{OH}$, and $-\mathrm{NH}$ - groups. The $-\mathrm{OH}$ group is hydrogen bonded with nearest nitrogen of $-\mathrm{NH}$ group present in the polymer chain through the $\mathrm{H}_{2} \mathrm{O}$ molecule present in the sample. So $-\mathrm{OH}$ absorption peak appears at about $3410 \mathrm{~cm}^{-1}$ as a broad peak. The peak at $1610 \mathrm{~cm}^{-1}$ is attributed to a mixture of the $\mathrm{C}=\mathrm{C}$ and $\mathrm{C}=\mathrm{N}$ stretching vibrations in the quinoid ring, whereas the peak at $1516 \mathrm{~cm}^{-1}$ is assigned to the $\mathrm{C}=\mathrm{C}$ stretching vibrations in the benzenoid ring. The peak appears at $2362 \mathrm{~cm}^{-1}$ which is the characteristics stretching band for $\mathrm{C}=\mathrm{C}=\mathrm{N}$ or $\mathrm{C}=\mathrm{C}=\mathrm{O}$ [10]. The peak at $1289 \mathrm{~cm}^{-1}$ is assigned to the $\mathrm{C}-\mathrm{N}$ stretching vibrations of the second aromatic amine, indicating the formation of a $\mathrm{C}-\mathrm{N}-\mathrm{C}$ structure in the polymer, whereas a peak at $2923 \mathrm{~cm}^{-1}$ is assigned to stretching vibrations of $\mathrm{C}=\mathrm{C}$ in phenyl ring [11]. The peak at $1140 \mathrm{~cm}^{-1}$ is ascribed to the stretching of the $\mathrm{C}-\mathrm{O}-\mathrm{C}$ linkages and further supports that the meta-aminophenol changed into PMAP. The peaks 


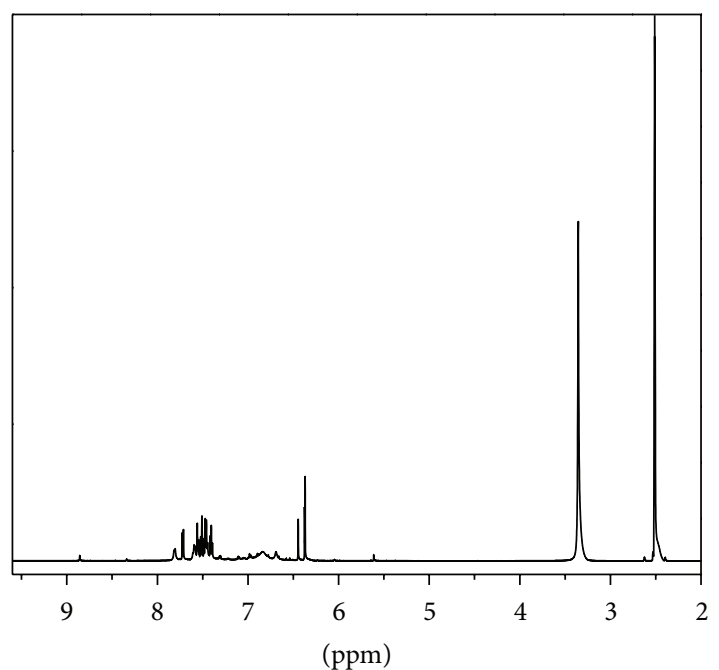

Figure $3:{ }^{1} \mathrm{H}$ NMR spectra of PMAP.

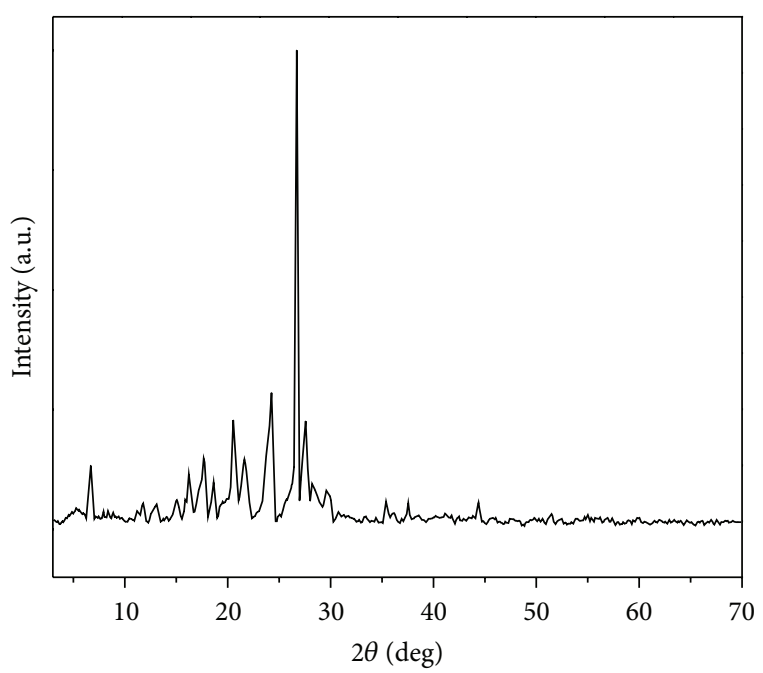

FIGURE 4: XRD pattern of PMAP.

between 900 and $600 \mathrm{~cm}^{-1}$ correspond to $\mathrm{C}-\mathrm{H}$ bending of an aromatic ring substitution.

3.4. ${ }^{1} H$ NMR Spectrum. The ${ }^{1} \mathrm{H}$ NMR spectra of PMAP recorded in $\mathrm{d}_{6}$-DMSO are shown in Figure 3. The protons present in $-\mathrm{CH}_{3}$ group of DMSO appear at $\delta 2.5$ and a broad peak appears at $\delta 3.4$ due to the protons present in the water molecule, which is present in the sample as well as in the solvent. The signals observed in the region of $\delta 6.2-7.8$ correspond to the protons of the aromatic rings. The terminal $-\mathrm{NH}_{2}$ groups in the compound give a very weak peak at $\delta 5.6$ as singlet [7]. Again other two singlet peaks are present at $\delta$ 9.1 and $\delta 9.36$ for the groups $-\mathrm{NH}-$ and hydrogen bonded $\mathrm{OH}$ group, respectively. This NMR result is in agreement with that of UV-VIS-NIR and FT-IR spectrum of PMAP.

3.5. XRD Analysis and Morphology. XRD pattern shows that the PMAP is highly crystalline as shown in Figure 4. It is

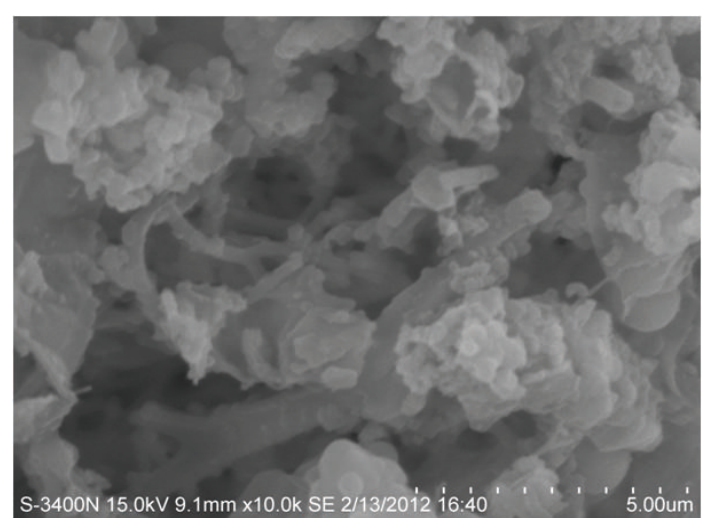

Figure 5: SEM micrograph of PMAP.

observed that discrete sharp and intense peaks are obtained in the diffractogram patterns. The sharp and high intense peaks appear at $27.57^{\circ}, 26.71^{\circ}, 24.21^{\circ}, 20.53^{\circ}$, and $6.65^{\circ}$ while three small sharp peaks are obtained at $44.35^{\circ}, 21.64^{\circ}$, and $17.67^{\circ}$, and other four small broad peaks of PMAP appear at $13.04^{\circ}$, $15.06^{\circ}, 35.42^{\circ}$, and $37.54^{\circ}$. It was suggested that the relatively broad reflection at about $26.71^{\circ}$ arises from the scattering perpendicular to the chain direction [12]. The higher degree of crystalline or more ordered structural pattern in PMAP is due to the presence of strong interchain hydrogen bonding as well as electrostatic (dipole-dipole) interactions involving both amine and phenolic groups present in the polymer chain. The d-spacing corresponding to the highest intense crystalline peak was determined by the Debye-Scherrer (powder) method using Bragg's relation [13], and it is found to be $3.34 \AA$. The mean crystallite size measured using Scherrer's formula [13] is found to be $61 \mathrm{~nm}$. The lattice strain value was calculated using Williamson-Hall (W-H) formula [14] and found to be $3.72 \times 10^{-3}$. The inter-chain separation length [13] corresponding to the highest intense crystalline peak is calculated and the value is $1.23 \AA$.

The micrograph reveals a flaky-like feature with few globular structures which is seen in the SEM image as shown in Figure 5. The photograph of PMAP reveals crystalline as well as amorphous regions. The crystalline regions with sharp edged particles and lamellar sides were found to be interspersed in the amorphous regions consisting of particles with no well-defined shapes. This result was supported from the XRD pattern of PMAP. The crystalline morphology may result from intramolecular hydrogen bonded with amino phenol units.

3.6. Thermogravimetric and Kinetic Analysis. The TGA and DTA thermograms of PMAP are shown in Figure 6 and exhibit a four-stage decomposition pattern. In the first stage mass loss (wt. 3.4\%) observed from 60 to $120^{\circ} \mathrm{C}$ indicates loss of water molecules, adsorbed moisture, free acids, and volatile molecules in polymer matrix. The second stage mass loss (wt. $13.4 \%$ ) which occurs in the range 150 to $190^{\circ} \mathrm{C}$ corresponds to the sublimation and removal of low molecular weight polymer/oligomer from the polymer matrix. The third mass loss (wt. 28.4\%) occurs in a temperature range starting from 


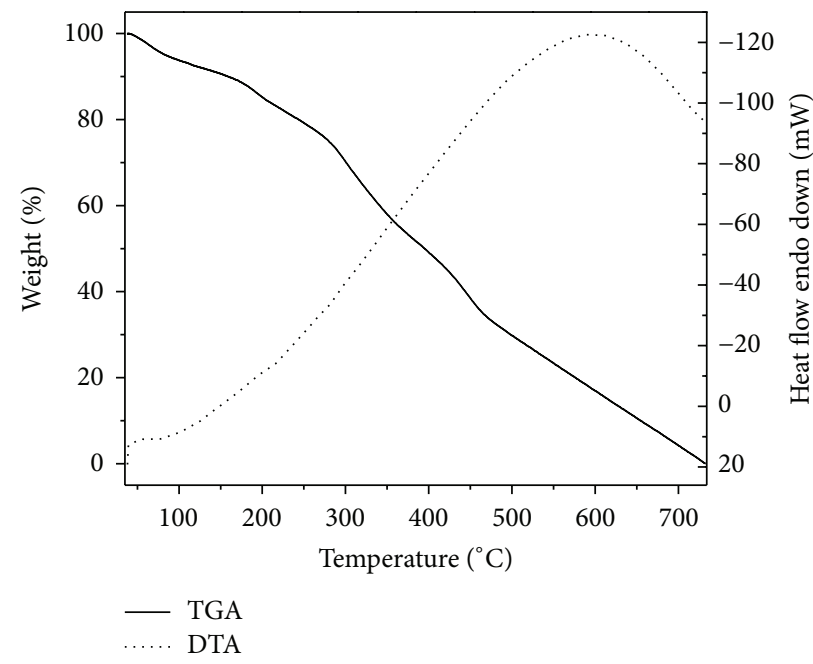

FIgURE 6: Thermogravimetric patterns TGA and DTA of PMAP as a function of temperature.

195 to $298^{\circ} \mathrm{C}$ because loss of dopant has been reported in the temperature range 200 to $300^{\circ} \mathrm{C}$ for polyaniline and its derivatives doped with different types of acidic dopants [15]. The fourth mass loss (wt. 57.5\%) step occurs between 390 and $463^{\circ} \mathrm{C}$. In this final step, the skeletal polymer chain structure decomposes after the elimination of the dopant from the polymer structure. The first exothermic peaks at 45 to $70^{\circ} \mathrm{C}$ in the DTA curve correlates to the loss of adsorbed $\mathrm{HCl}$ and moisture in the TG study. The two endothermic peak at 198 and $285^{\circ} \mathrm{C}$ can be attributed to the morphological changes and disruption of inter- and intra-molecular hydrogen bonding and loss of dopants. The second broad exotherm denoting the final degradation step occurs around $470^{\circ} \mathrm{C}$.

Thermal degradation kinetic parameter was evaluated from TGA curves using Coats-Redfern [16] and Broido's [17] methods. For the sake of calculations and to know the nature of the decomposition, the complete thermogram was divided into distinct sections according to their degradation processes. Coats-Redfern (CR) relation is

$$
\log \left(\frac{-\log (1-\alpha)}{T^{2}}\right)=\left(\log \left(\frac{A R}{\beta E_{a}}\right)\right)-\left(\frac{E_{a}}{2.303 R T}\right)
$$

where $\alpha$ is the fraction of sample decomposed at temperature $T, T$ is the derivative peak temperature, $A$ is the frequency factor, $\beta$ is the heating rate, $E_{a}$ is the activation energy, and $R$ is the gas constant. Mathematical expression of Broido's (BR) method is

$$
\log (-\log (1-\alpha))=-\left(\frac{E_{a}}{2.303 R T}\right)\left(\left(\frac{1}{T}\right)+K\right),
$$

where $(1-\alpha)$ is the fraction of number of initial molecules not yet decomposed and $T$ is the peak temperature of derivative curve of TGA. The plots of $\log \left(-\log (1-\alpha) / T^{2}\right)$ versus $1 / T$ (CR) and $\log (-\log (1-\alpha))$ versus $1 / T$ (BR) for PMAP are shown in Figures 7 and 8, respectively. These plots follow

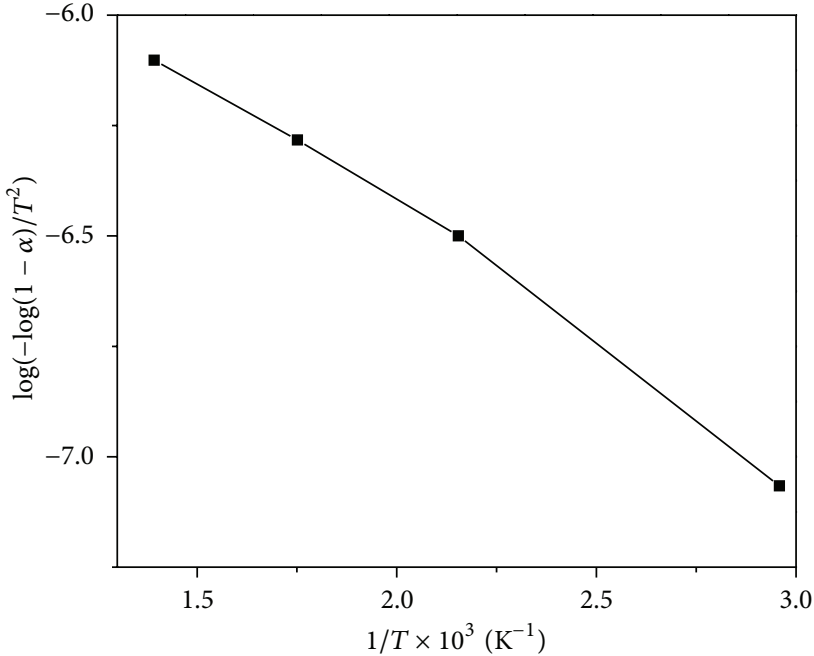

Figure 7: Plot of $\log \left(-\log (1-\alpha) / T^{2}\right)$ versus $1 / T$ using CoatsRedfern method for PMAP.

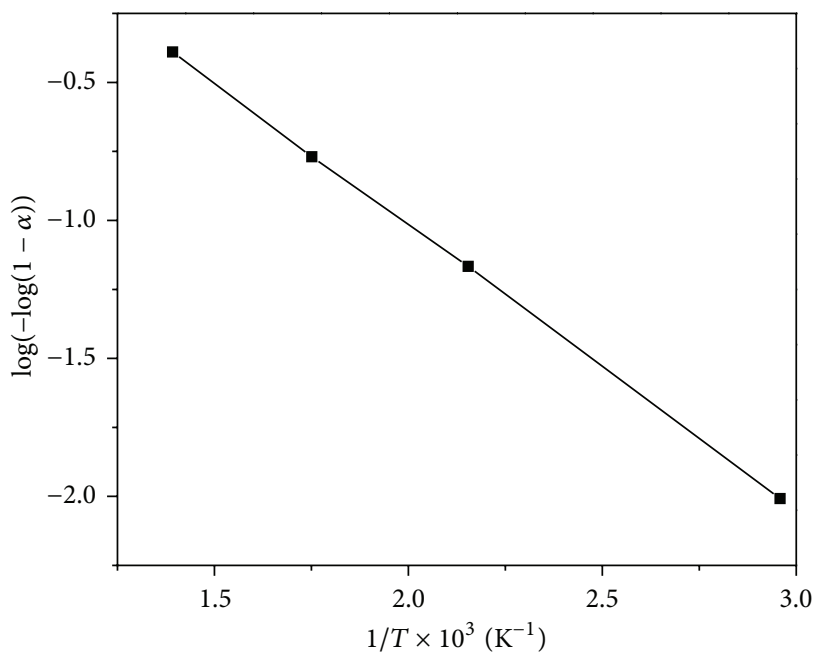

Figure 8: Plot of $\log (-\log (1-\alpha))$ versus $1 / T$ using Broido's method for PMAP.

straight line whose slopes give the activation energy $\left(E_{a}\right)$ of degradation process. The activation energies of CR and BR methods are 11.87 and $19.74 \mathrm{~kJ} / \mathrm{mol}$, respectively.

3.7. AC Conductivity. It is a well-known fact that frequencydependent complex conductivity in case of disordered materials such as polymers can arise from interfacial polarization at contacts, grain boundaries, and other inhomogeneities present in the sample. The ac conductivity of insulator/ conductor polymers has been modelled by a resistancecapacitance network, where the conducting dispersants are represented as a resistors and the dielectric constant of the insulating matrix is represented by capacitors [18].

The complex impedance plots for PMAP measured at temperatures ranging from 303 to $383 \mathrm{~K}$ in the frequency region $20 \mathrm{~Hz}$ to $10^{6} \mathrm{~Hz}$ are shown in Figure 9. The point of 


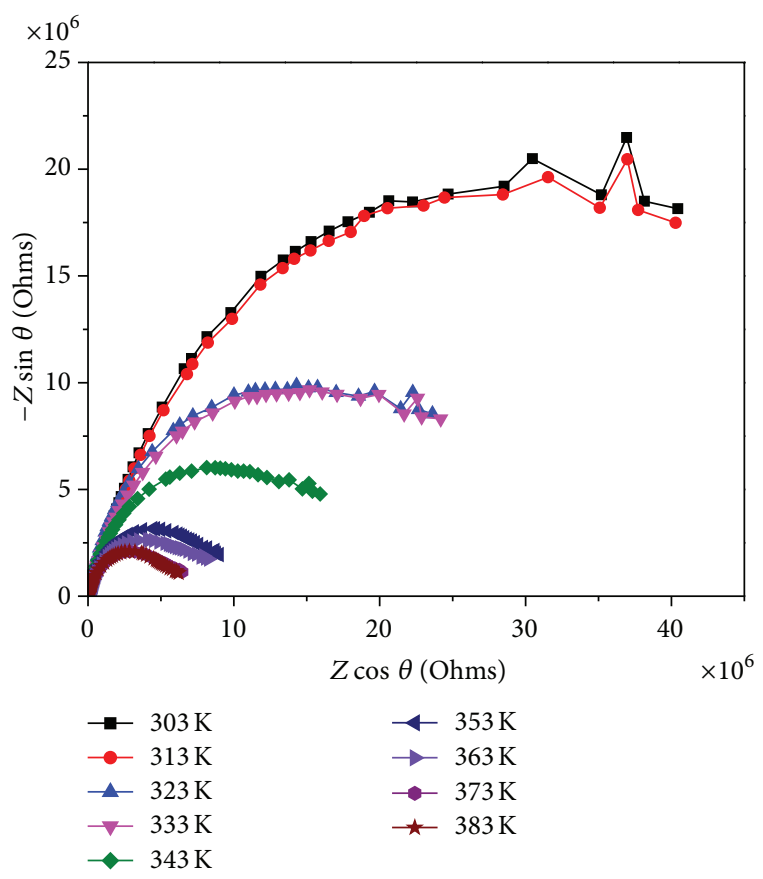

FIgURE 9: Complex impedance plots of PMAP at different temperatures.

intersection of the impedance plot on the real axis gives the bulk resistance of the polymer. It can be seen from Figure 9 that as the temperature is increased, the point of intersection is shifted towards the origin. In other words, as the temperature increases the intersection is found to shift towards higher frequency. Hence, it is evident that the bulk resistance of the polymer decreases with the increase in temperature, resulting in the enhancement of electrical conductivity at higher temperatures. The temperature-dependant conductivity value for PMAP reveals that the conductivity increases with temperature. The increase in conductivity with increase in temperature is the characteristic of "thermal-activated behavior" due to the increased efficiency of charge transfer between polymer chains with the increase in temperature [19].

The temperature-dependent conductivity data obtained for PMAP over the temperature range 303 to $383 \mathrm{~K}$ are shown in Figure 10, in the form of plots of $\log \sigma T$ versus $1000 / T$ where $\sigma$ denotes the conductivity and $T$ the absolute temperature. It is interesting to note from Figure 10 that the value of electrical conductivity $(\sigma)$ increases with increasing temperature as in the case of polymers obeying the Arrhenius relationship,

$$
\sigma T=\sigma_{0} \exp \left(-\frac{E_{a}}{K T}\right)
$$

where $\sigma_{0}$ denotes the preexponential factor, $E_{a}$ denotes the energy of activation for conduction, and $K$ is the Boltzmann constant. The activation energy was calculated from the slope of the observed linear plot drawn by the least square method and was found to be $0.11 \mathrm{eV}$. The low activation energy for this polymer is attributed to the enhancement of the electronic

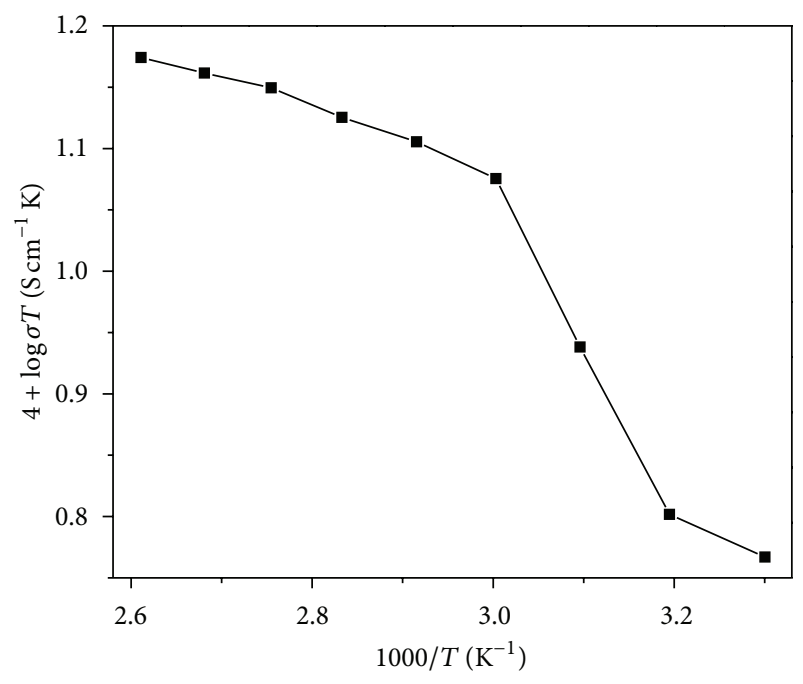

FIgURE 10: Temperature-dependent AC conductivity data of PMAP at different temperatures.

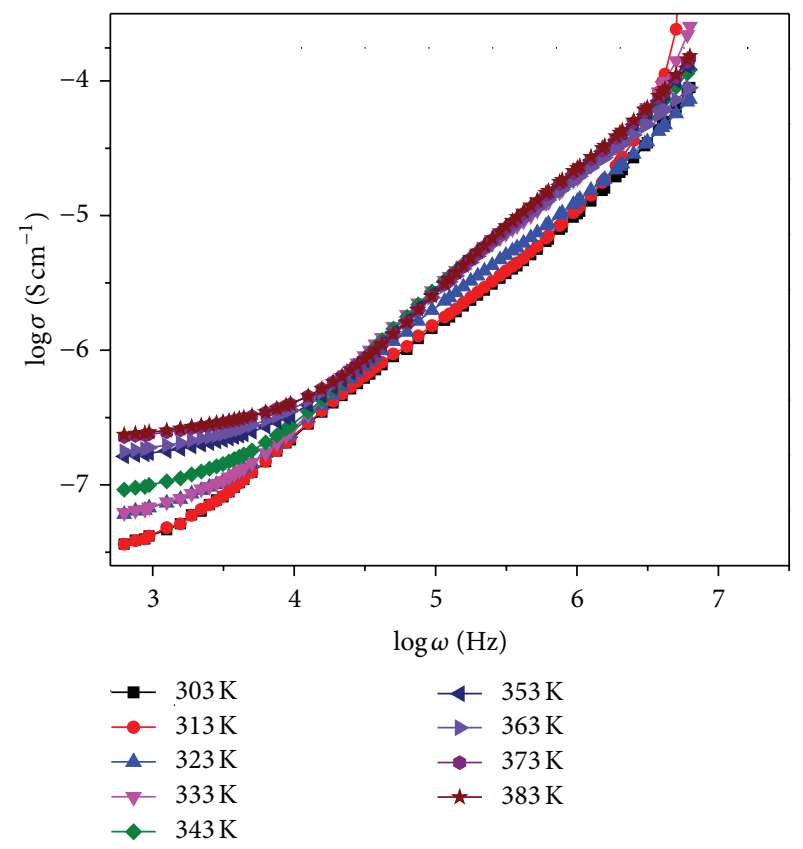

FIgURE 11: Frequency-dependent AC conductivity (log $\sigma$ versus $\log \omega)$ plots of PMAP at different temperatures.

jump between the localized states as found for other materials [20].

To investigate the nature of frequency-dependent $\mathrm{AC}$ conductivity, the plot of $\log (\sigma T)$ versus $\log (\omega)$ with in a frequency range $20 \mathrm{~Hz}$ to $10^{6} \mathrm{~Hz}$ over the temperature range 303 to $383 \mathrm{~K}$ is drawn and the results are shown in Figure 11. A frequency-dependant electrical conductivity $\sigma_{\mathrm{ac}}(\omega)$ has been observed in many semiconductors, insulators, and also in inorganic and polymeric organic materials. The charge transport measurements in these disordered solids will provide information about the electronic structure of 
TABLE 1: Frequency exponent $(S)$, DC conductivity and relaxation time $(\tau)$ of PMAP at different temperatures.

\begin{tabular}{lccc}
\hline Temperature $(\mathrm{K})$ & $S$ & $\begin{array}{c}\text { DC conductivity } \\
\times 10^{-7}\left(\mathrm{~S} \mathrm{~cm}^{-1}\right)\end{array}$ & $\tau(\mu \mathrm{s})$ \\
\hline 303 & 0.93 & 0.56 & 0.80 \\
313 & 0.90 & 0.81 & 0.78 \\
323 & 0.84 & 1.51 & 0.67 \\
333 & 0.83 & 1.62 & 0.62 \\
343 & 0.81 & 2.51 & 0.39 \\
353 & 0.79 & 3.34 & 0.33 \\
363 & 0.76 & 3.80 & 0.28 \\
373 & 0.75 & 4.03 & 0.27 \\
383 & 0.74 & 4.12 & 0.25 \\
\hline
\end{tabular}

the materials. The disorder in atomic configuration and/or composition causes localization of electronic states or groups of states within the material. Since the charge carriers are localized, AC impedance techniques are used to examine such behaviours [19]. The frequency dependence of the alternative current (ac) conductivity follows a power law behavior. $\sigma^{\prime}(\omega)$ or the total AC conductivity can be represented by the following equation:

$$
\sigma^{\prime}(\omega)=\sigma_{0}+\sigma_{\mathrm{ac}}(\omega)=\sigma_{\mathrm{dc}}+A \omega^{S},
$$

where $\omega$ is the angular frequency, $\sigma_{\mathrm{dc}}$ is the independent frequency conductivity or DC conductivity, $A$ is the constant dependent on temperature $T$, and $S$ is an exponent dependent on both frequency and temperature with values in the range 0 to 1 . This type of behaviour was noted by Jonscher, who called it the "Universal Dynamic Response" (UDR) [21] because of a wide variety of materials that displayed such behavior. From Figure 11, a linear increase in conductivity with frequency is noticed and the value of the frequency exponent $S$, estimated from the slope of the $\log (\sigma T)$ versus $\log (\omega)$, which are fully consistent with the power law. The dc conductivity value has also been calculated by extrapolating the ac conductivity data to zero frequency region and both $S$ and dc conductivity values are presented in Table 1 . The dc conductivity increases as increasing with temperature. The temperature dependence of $S$ for PMAP (Table 1) shows clearly that $S$ decreases with the increase in the temperature. According to the Quantum Mechanical Tunneling (QMT) model [4] the exponent $S$ is almost equal to 0.8 and increases slightly or does not increase with the increase in the temperature. Therefore, the QMT model does not allow being in agreement with the values which were obtained for $S$ in PMAP. According to the Overlapping Large Polaron Tunneling (OLPT) model [22], the exponent $S$ is both temperature- and frequency-dependent. $S$ decreases with increasing temperature from unity at room temperature to a minimum value at a certain temperature, and then it increases with increasing temperature. Therefore, the OLPT model is also not applicable to our results in this temperature range. According to the Correlated Barrier Hopping $(\mathrm{CBH})$ model, the values of the frequency exponent $S$ extend from 0.6 to 1 and prove to decrease with the increase in the temperature [23]. This model is in good agreement with our experimental results suggesting that the electrical AC conduction mechanism of PMAP at investigated temperature (303 to $383 \mathrm{~K}$ ) can be explained by charge-carrier hops between sites over the potential barrier $W_{M}$ separating them. The frequency exponent $S$ for such model is given by the following equation [24]:

$$
S=1-\frac{6 K_{B} T}{\left(W_{M}-K T \ln (\omega \tau)\right)},
$$

where $W_{M}$ is the height of maximum barrier (or binding energy) and $\tau$ is the relaxation time. $W_{M}$ is calculated from the linear line slope which is between $1-S$ and $T$. A value of $W_{M}=0.19 \mathrm{eV}$ is obtained for PMAP.

3.8. Dielectric Properties. The dielectric properties of conducting polymers are one of the main focus points of research because of their novel technological applications. It is well established that the polymers, as dielectric materials, are excellent host matrices and also provide environmental and chemical stability [25].

The real part $\left(\varepsilon^{\prime}\right)$ of the dielectric function $\left(\varepsilon^{*}\right)$ has been calculated from the measured values of capacitance using the following formula:

$$
\varepsilon^{\prime}=\frac{C d}{\varepsilon_{0} A},
$$

where $C$ is the measured capacitance of the sample, $d$ is the thickness of the sample, $A$ is the area of the pellet, and $\varepsilon_{0}$ is the permittivity of free space. The imaginary part $\left(\varepsilon^{\prime \prime}\right)$ of the complex impedance has been measured as

$$
\varepsilon^{\prime \prime}=\varepsilon^{\prime} \tan \delta,
$$

where $\tan \delta$ is the dissipation factor. Figures $12(\mathrm{a})$ and $12(\mathrm{~b})$ depict the variation of dielectric constant $\left(\varepsilon^{\prime}\right)$ and dielectric loss $\left(\varepsilon^{\prime \prime}\right)$ as a function of frequency for PMAP, respectively, in the temperature range of 303 to $383 \mathrm{~K}$. The dielectric constant of the PMAP decreases moderately with frequency. As the frequency increases, the charge carriers migrating through the dielectric get trapped against a defect site and induce an opposite charge in its vicinity, as a result of which they slow down and the value of dielectric constant decreases moderately [26]. It is interesting to observe that the dependence of loss factor with the frequency of PMAP (Figure 12(b)) which decreases sharply with increase in frequency. The larger value of the loss factor or dielectric loss at low frequency could be due to the mobile charges within the polymer matrix. At high frequency, periodic field reversal is so fast that there is no excess ion diffusion in the direction of electric field [27]. Polarization due to charge accumulation decreases, leading to the decrease in the value of loss factor. Generally it is believed in dielectric analysis that the high frequency dielectric constant is mainly associated with dipolar relaxation, whereas at lower frequency and higher temperature, the contributions of interfacial polarization and dc conductivity become more significant in both $\varepsilon^{\prime}$ and $\varepsilon^{\prime \prime}$. 


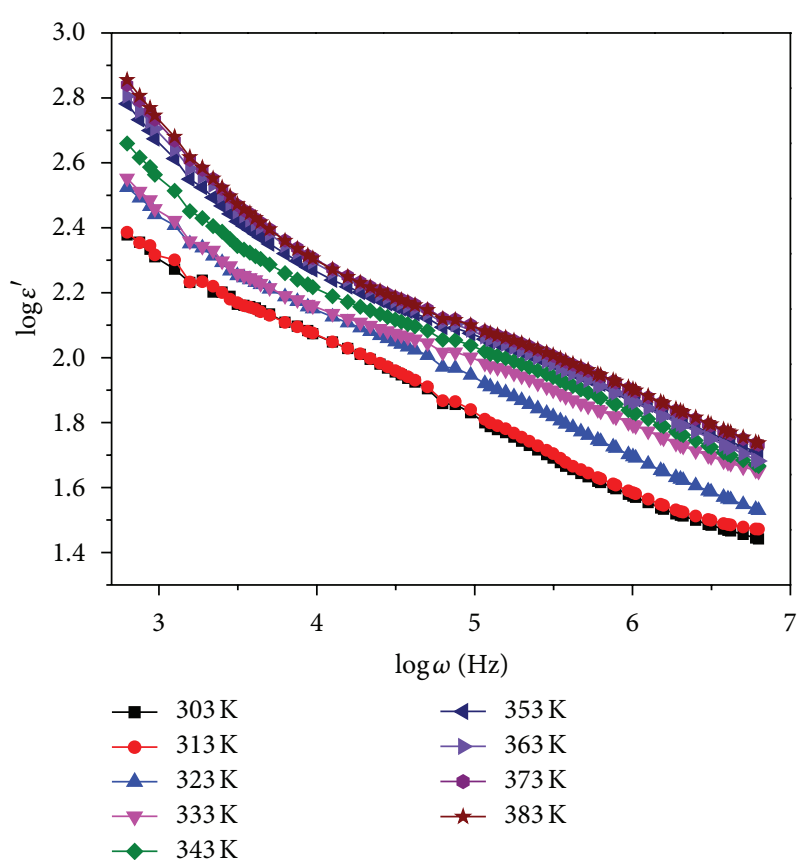

(a)

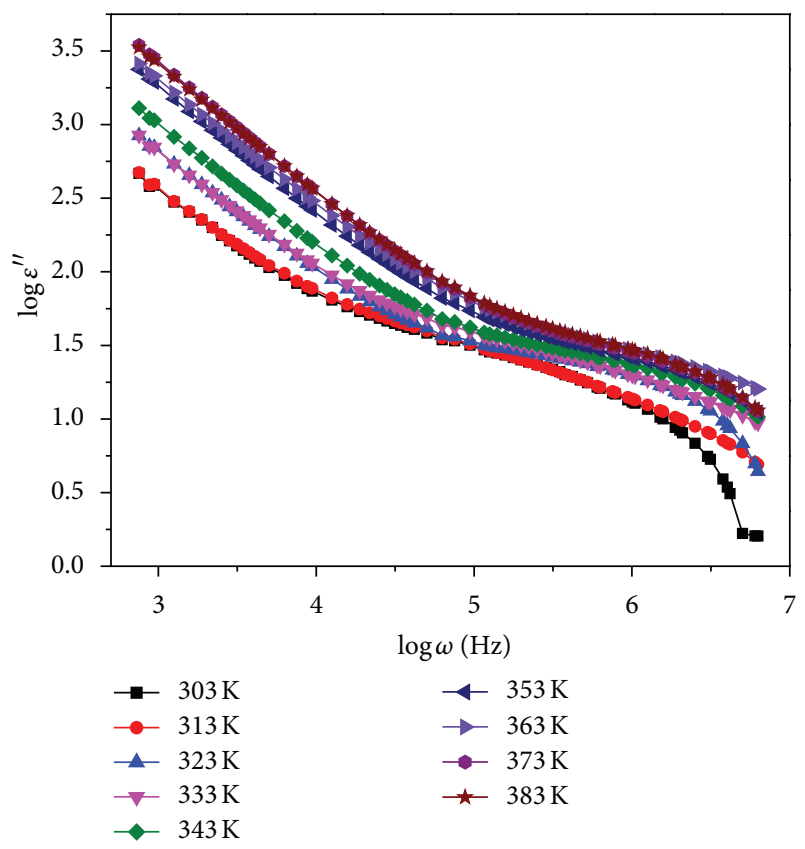

(b)

FIGURE 12: (a) Logarithmic plots of dielectric constant as a function of frequency for PMAP. (b) Logarithmic plots of dielectric loss as a function of frequency for PMAP.

Figures 13(a) and 13(b) show the isothermal and isochronal dependence of $M^{\prime \prime}$ on the frequency and temperature for PMAP, respectively. It exhibits two peaks in both isothermal and isochronal plots, indicating clearly two different relaxation processes. The appearance of two relaxation peaks may be considered to be due to the two-phase structure in the polymer. The lower frequency peak can be

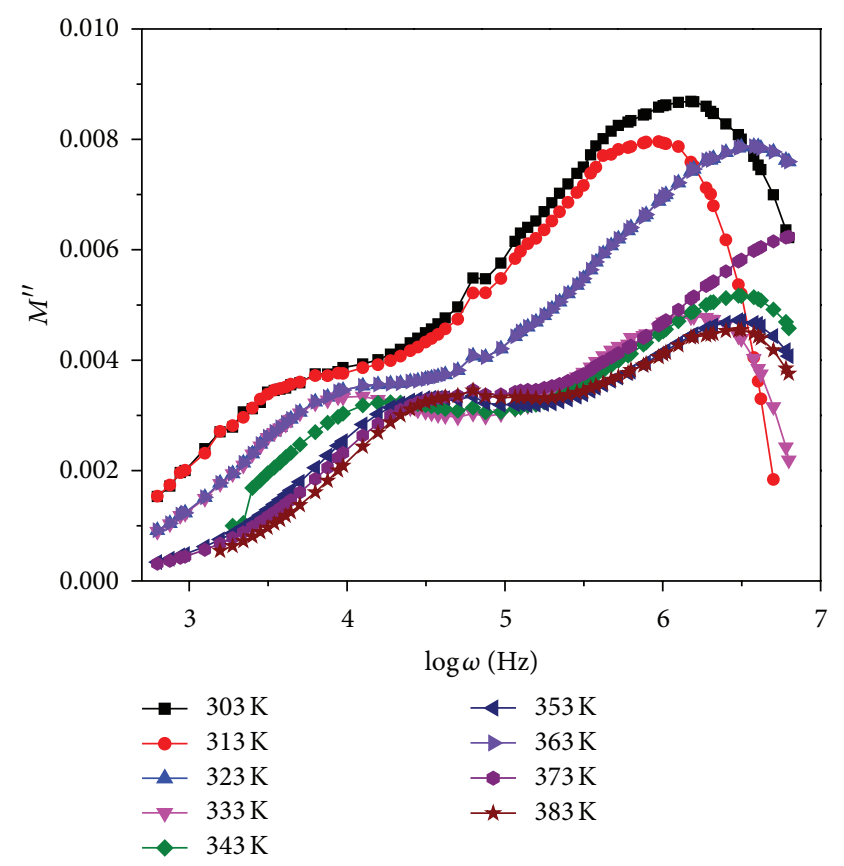

(a)

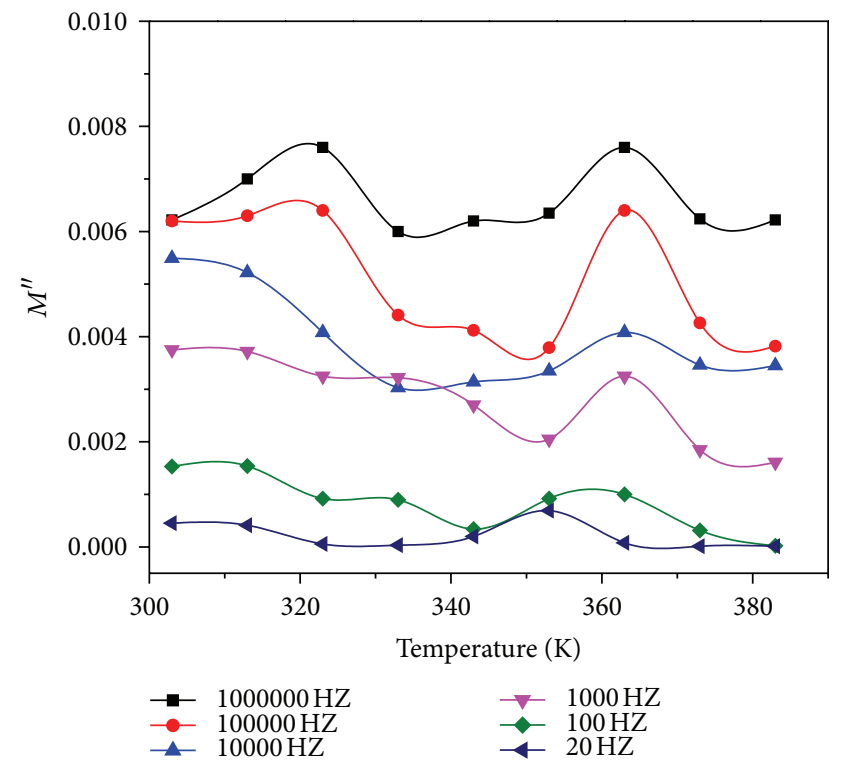

(b)

FIGURE 13: (a) Isothermal graphs of imaginary part of electric modulus for PMAP. (b) Isochrones graphs of imaginary part of electric modulus for PMAP.

attributed to the phase of oxidized repeat units (quinoid) and the higher frequency peak to the phase of reduced repeat units (benzenoid) of PMAP [28]. The positions of the two relaxations peaks do not change strongly with temperature. The position of the characteristic relaxation frequency, $\omega_{\max }$, in plots of $M^{\prime \prime}$ versus $\log \omega$ depends upon the conductivity relaxation time, according to the expression $\omega_{\max }=1 / 2 \pi \tau$. Relaxation time $(\tau)$ is a temperature-dependent factor and it decreases as the temperature increases as shown in Table 1. 


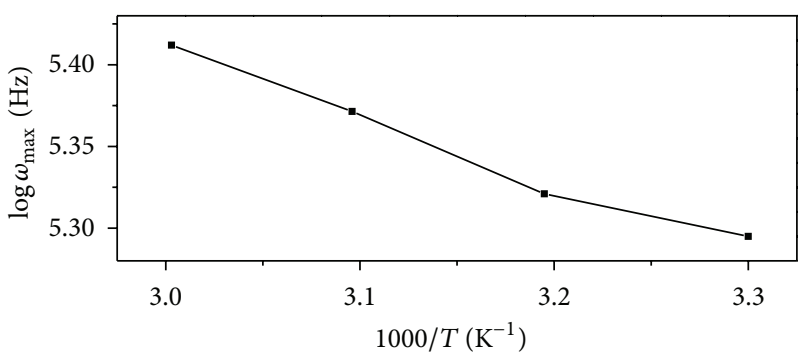

(a)

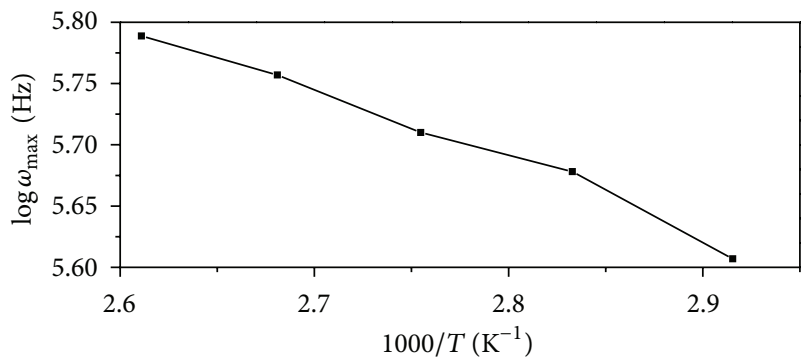

(b)

Figure 14: Arrhenius plot of relaxation frequencies with temperature corresponding to the two peaks observed from the plots of $M^{\prime \prime}$ versus $\log \omega$.

It is observed that the isothermal peaks (Figure 14(a)) shift towards higher frequency when temperature is increased and similarly the isochronal peaks (Figure 13(b)) shift towards higher temperature when the frequency is increased. Therefore, the activation energy $\left(E_{a}\right)$ of these relaxation processes can be estimated from plots of $\log \omega_{\max }$ versus $1 / T$ (from isothermal plots) or $\log \omega$ versus $1 / T_{\max }$ (from isochronal plots). These plots are straight lines in accordance with the Arrhenius equation. The activation energy value estimated from isothermal plot (Figure 14) for first and second relaxation processes is found to be 0.07 and $0.1 \mathrm{eV}$, respectively. The height of the peak corresponding to the relaxation of the reduced units (higher frequency) is significantly lower than the peak corresponding to the oxidized unit.

The complex plane diagram of the electric modulus for PMAP is illustrated in Figure 15. PMAP clearly exhibits two arcs corresponding to two relaxation processes at different temperatures ranging from 303 to $313 \mathrm{~K}$, as also observed in $M^{\prime \prime}(\log \omega)$ or $M^{\prime \prime}(T)$. The arcs initiate from the origin and spread to different values of $M^{\prime}$ depending upon the temperature. These curves do not form semicircles, which could correspond to the idealized Debye model with double relaxation time. They rather possess a shape of deformed arcs with their centres positioned below the horizontal axis.

The reduced plots of $M^{\prime \prime} / M_{\max }^{\prime \prime}$ versus $\log \left(\omega / \omega_{\max }\right)$ at different temperatures provide additional information regarding the molecular heterogeneity of the polymer. Figure 16 illustrates reduced plot for PMAP and shows that the distribution of relaxation times is nearly the same over the temperature range from 303 to $383 \mathrm{~K}$. The shape of the reduced modulus spectra at different temperatures clearly signifies

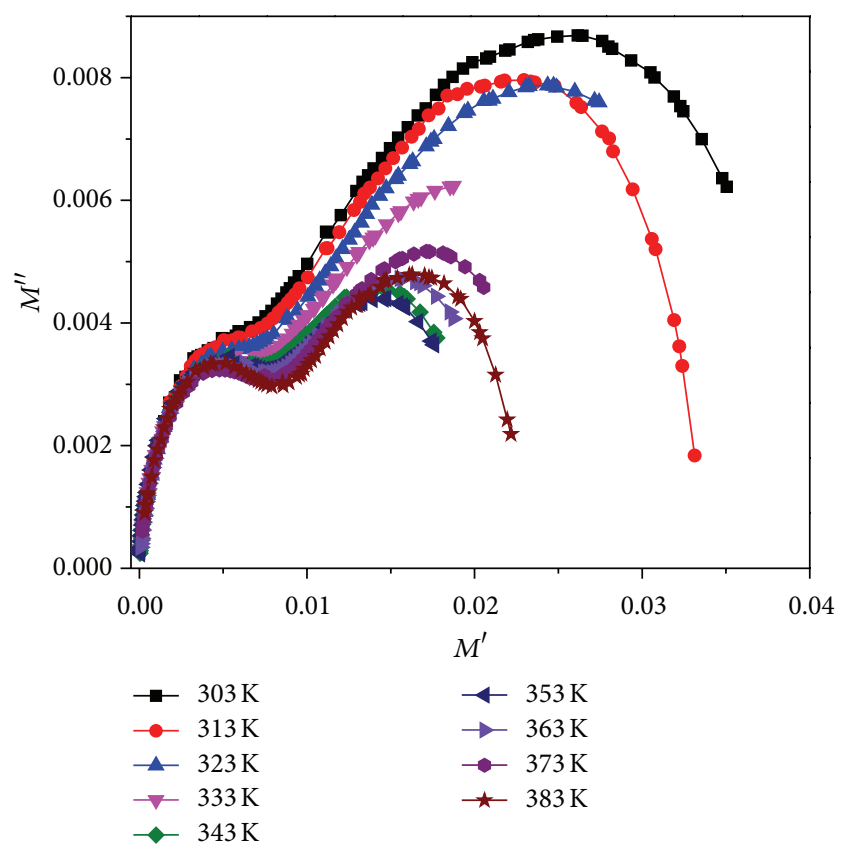

FIGURE 15: Complex planes for the electric modulus of PMAP at different temperatures.

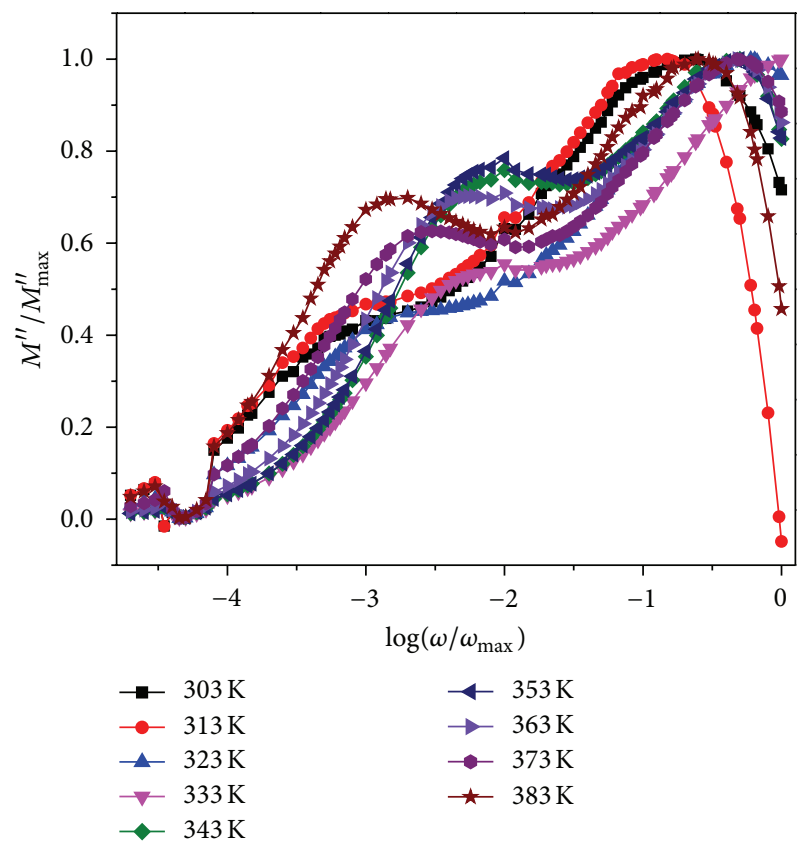

FIgURE 16: Normalized plots of $M^{\prime \prime} / M_{\max }^{\prime \prime}$ versus $\log \left(\omega / \omega_{\max }\right)$ at different temperatures of PMAP.

the temperature independence of the distribution of relaxation times (DRT), in view of the heterogeneous nature of the present polymer. The physical significance of the temperature-independent DRT is that the distribution of conductivities in the polymer is temperature independent [29]. 


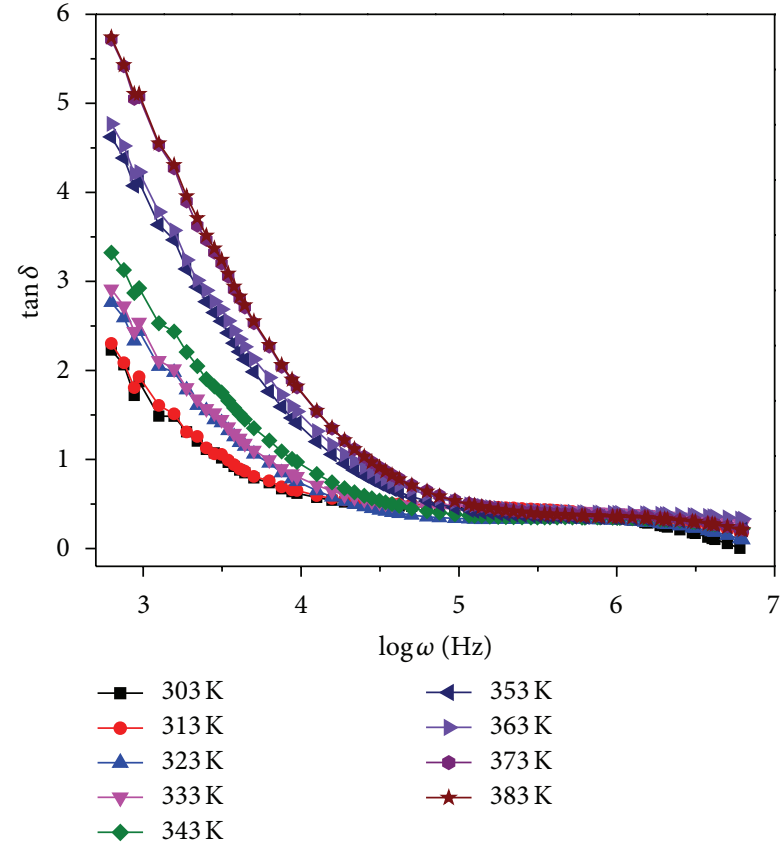

(a)

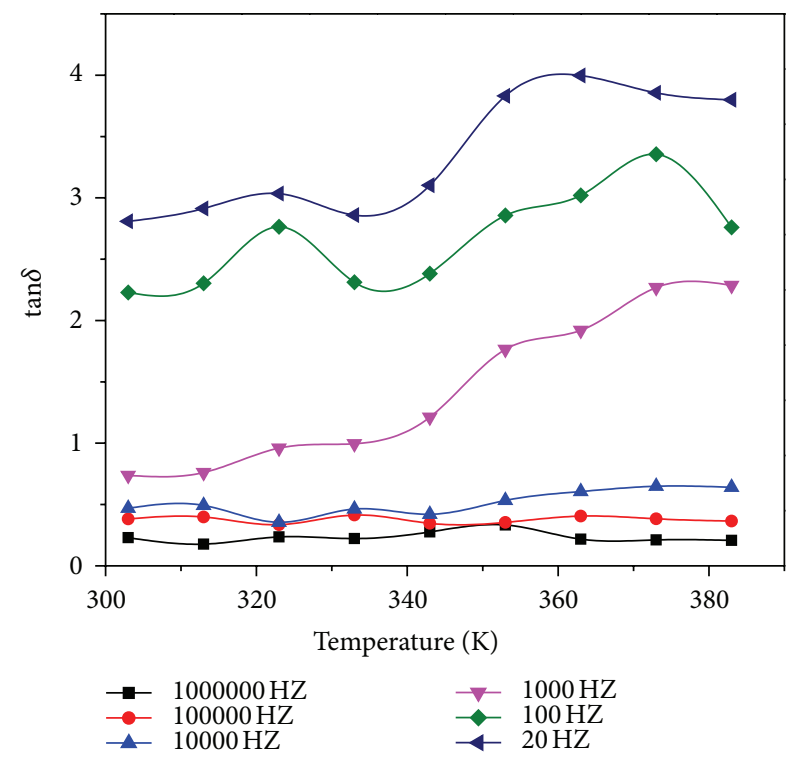

(b)

FIGURE 17: (a) Frequency dependence of dissipation factor of PMAP. (b) Temperature dependence of dissipation factor of PMAP.

The dissipation factor, $\tan \delta$, recorded as a function of frequency and temperature for PMAP is shown in Figures 17 (a) and 17(b), respectively. The loss tangent decreases with increasing frequency. As noted earlier, high loss at low frequency in loss tangent may be attributed to DC conduction losses as in the case of dielectric constant and dielectric loss. At higher frequencies PMAP exhibits almost zero dielectric loss which suggests that this polymer is lossless material at frequencies beyond $10^{5} \mathrm{~Hz}$. The frequency and temperature dependence loss tangent, however, revealed two relaxation peaks as shown in Figures 13(a) and 13(b). The first relaxation being the low temperature process may arise due to inhomogeneities present in the polymer. The second relaxation process at higher temperature is attributed to the increase in the barrier potential resulting from the decrease in polymer conjugation due to increased movement of the chain segments [30].

\section{Conclusion}

Poly Meta Aminophenol has been successfully synthesized by chemical oxidative method at $0-3^{\circ} \mathrm{C}$ and characterized by different spectroscopic techniques. The characterization by UV-VIS-NIR, FT-IR, and ${ }^{1} \mathrm{H}$ NMR suggests the formation of quinoid and benzenoid rings which confirmed the formation of polymer. The XRD and SEM studies reveal that the morphology of PMAP is partially crystalline and amorphous nature. It was observed from thermal analysis that PMAP exhibits four-step decomposition patterns. The frequencydependent electrical parameters, such as impedance, AC conductivity, dielectric constant, dielectric loss, and electric modulus for PMAP, have been investigated within the range of $20 \mathrm{~Hz}$ to $10^{6} \mathrm{~Hz}$ over the temperature range of 303 to $383 \mathrm{~K}$. Ac conductivity increases moderately with temperature and obeys the power law of frequency. The temperature variation of frequency exponent suggests that the ac conductivity can be described by $\mathrm{CBH}$ mechanism. The dielectric constant and dielectric loss decrease with an increase in frequency. The electric modulus and dissipation factor recorded as a function of frequency and temperature display two relaxation processes. At higher frequencies PMAP exhibits low dielectric loss, which suggests that PMAP is lossless material at frequencies beyond $10^{5} \mathrm{~Hz}$.

\section{Conflict of Interests}

The authors declare that there is no conflict of interests regarding the publication of this paper.

\section{References}

[1] C. V. Bouanga, K. Fatyeyeva, P.-Y. Baillif et al., "Study of dielectric relaxation phenomena and electrical properties of conductive polyaniline based composite films," Journal of NonCrystalline Solids, vol. 356, no. 11-17, pp. 611-615, 2010.

[2] E. Hür, G. Bereket, B. Duran, D. Özdemir, and Y. Şahin, "Electropolymerization of $\mathrm{m}$-aminophenol on mild steel and its corrosion protection effect," Progress in Organic Coatings, vol. 60, no. 2, pp. 153-160, 2007.

[3] M. Nadeem, M. J. Akhtar, and M. N. Haque, "Increase of grain boundary resistance with time by impedance spectroscopy in $\mathrm{La}_{0.50} \mathrm{Ca}_{0.50} \mathrm{MnO}_{3+\delta}$ at $77 \mathrm{~K}$," Solid State Communications, vol. 145, no. 5-6, pp. 263-266, 2008.

[4] U. Akgul, Z. Ergin, M. Sekerci, and Y. Atici, "AC conductivity and dielectric behavior of $\left.[\mathrm{Cd} \text { (phen })_{2}(\mathrm{SCN})_{2}\right]$," Vacuum, vol. 82, no. 3, pp. 340-345, 2007.

[5] R. Singh, J. Kumar, R. K. Singh, A. Kaur, R. D. P. Sinha, and N. P. Gupta, "Low frequency ac conduction and dielectric relaxation behavior of solution grown and uniaxially stretched 
poly(vinylidene fluoride) films," Polymer, vol. 47, no. 16, pp. 5919-5928, 2006.

[6] A. Falcou, A. Duchêne, P. Hourquebie, D. Marsacq, and A. Balland-Longeau, "A new chemical polymerization process for substituted anilines: application to the synthesis of $\operatorname{poly}(\mathrm{N}$ alkylanilines) and poly(o-alkylanilines) and comparison of their respective properties," Synthetic Metals, vol. 149, no. 2-3, pp.115122, 2005.

[7] P. Kar, N. C. Pradhan, and B. Adhikari, "A novel route for the synthesis of processable conducting poly(m-aminophenol)," Materials Chemistry and Physics, vol. 111, no. 1, pp. 59-64, 2008.

[8] A. Elmansouri, A. Outzourhit, A. Lachkar et al., "Influence of the counter ion on the properties of poly (o-toluidine) thin films and their Schottky diodes," Synthetic Metals, vol. 159, no. 3-4, pp. 292-297, 2009.

[9] P. S. Rao and D. N. Sathyanarayana, "Synthesis of electrically conducting copolymers of $o-/ m$-toluidines and $o-/ m$-amino benzoic acid in an organic peroxide system and their characterization," Synthetic Metals, vol. 138, no. 3, pp. 519-527, 2003.

[10] P. Kar, N. C. Pradhan, and B. Adhikari, "Induced doping by sodium ion in poly(m-aminophenol) through the functional groups," Synthetic Metals, vol. 160, no. 13-14, pp. 1524-1529, 2010.

[11] C. Chen, C. Sun, and Y. Gao, "Electrosynthesis of poly(anilineco-p-aminophenol) having electrochemical properties in a wide pH range," Electrochimica Acta, vol. 53, no. 7, pp. 3021-3028, 2008.

[12] D. T. Seshadri and N. V. Bhat, "Structural and electrical properties of crystals of substituted polyaniline," Journal of Polymer Science B, vol. 45, no. 10, pp. 1127-1137, 2007.

[13] S. Bhadra and D. Khastgir, "Determination of crystal structure of polyaniline and substituted polyanilines through powder Xray diffraction analysis," Polymer Testing, vol. 27, no. 7, pp. 851857, 2008.

[14] K. Venkateswarlu, A. Chandra Bose, and N. Rameshbabu, "Xray peak broadening studies of nanocrystalline hydroxyapatite by WilliamsonHall analysis," Physica B, vol. 405, no. 20, pp. 4256-4261, 2010.

[15] C. Sivakumar, T. C. Wen, A. Gopalan, and H. Teng, "Electroactive conducting blends of poly(o-toluidine) and poly(vinylidene fluoride) and characterisation," Synthetic Metals, vol. 132, no. 3, pp. 219-226, 2003.

[16] A. W. Coats and J. P. Redfern, "Kinetic parameters from thermogravimetric data," Nature, vol. 201, no. 4914, pp. 68-69, 1964.

[17] B. A. Broido, "A Simple, sensitive graphical method of treating thermogravimetric analysis data," Journal of Polymer Science A, vol. 7, no. 10, pp. 1761-1773, 1969.

[18] Ramesh Patil, A. S. Roy, K. R. Anilkumar, K. M. Jadhav, and Shrikant Ekhelikar, "Dielectric relaxation and ac conductivity of polyaniline-zinc ferrite composite," Composites B, vol. 43, no. 8, pp. 3406-3411, 2012.

[19] P. Chithra Lekha, S. Subramanian, and D. Pathinettam Padiyan, "Investigation of pseudocapacitance effect and frequency dependence of ac impedance in Polyaniline-polyoxometalate hybrids," Journal of Materials Science, vol. 44, no. 22, pp. 60406053, 2009.

[20] M. A. M. Seyam, A. E. Bekheet, and A. Elfalaky, "AC conductivity and dielectric properties of In2S3 films," European Physical Journal, vol. 16, no. 2, pp. 99-104, 2001.

[21] A. K. Jonscher, "The "universal" dielectric response," Nature, vol. 267, no. 5613, pp. 673-679, 1977.
[22] A. R. LONG, "Frequency-dependent loss in amorphous semiconductors," ADV PHYS, vol. 31, no. 5, pp. 553-637, 1982.

[23] J. T. Gudmundsson, H. G. Svavarsson, S. Gudjonsson, and H. P. Gislason, "Frequency-dependent conductivity in lithiumdiffused and annealed GaAs," Physica B, vol. 340-342, pp. 324328, 2003.

[24] A. Kahouli, A. Sylvestre, F. Jomni, B. Yangui, and J. Legrand, "Experimental and theoretical study of AC electrical conduction mechanisms of semicrystalline parylene C thin films," Journal of Physical Chemistry A, vol. 116, no. 3, pp. 1051-1058, 2012.

[25] N. Singh and P. K. Khanna, "In situ synthesis of silver nanoparticles in polymethylmethacrylate," Materials Chemistry and Physics, vol. 104, no. 2-3, pp. 367-372, 2007.

[26] A. Qureshi, A. Mergen, M. S. Eroĝlu, N. L. Singh, and A. Güllüoĝlu, "Dielectric properties of polymer composites filled with different metals," Journal of Macromolecular Science A, vol. 45, no. 6, pp. 462-469, 2008.

[27] P. B. Bhargav, B. A. Sarada, A. K. Sharma, and V. V. R. N. Rao, "Electrical conduction and dielectric relaxation phenomena of PVA based polymer electrolyte films," Journal of Macromolecular Science A, vol. 47, no. 2, pp. 131-137, 2010.

[28] S. Banerjee and A. Kumar, "Relaxation and charge transport phenomena in polyaniline nanofibers: swift heavy ion irradiation effects," Journal of Non-Crystalline Solids, vol. 358, no. 22, pp. 2990-2998, 2012.

[29] S. A. Suthanthiraraj and Y. D. Premchand, "An evaluation of silver ionic transport in the mixed system (1-x) $\mathrm{CuI}-\mathrm{x} \cdot \mathrm{Ag}_{2} \mathrm{MoO}_{4}$ $(0.15 \leq \mathrm{x} \leq 0.6)$," Journal of Solid State Chemistry, vol. 170, no. 1 , pp. 142-153, 2003.

[30] A. N. Mallya, G. S. Yashavanth Kumar, Rajeev Ranjan, and P. C. Ramamurthy, "Dielectric relaxations above room temperature in DMPU derived polyaniline film," Physica B, vol. 407, no. 18, pp. 3828-3832, 2012. 

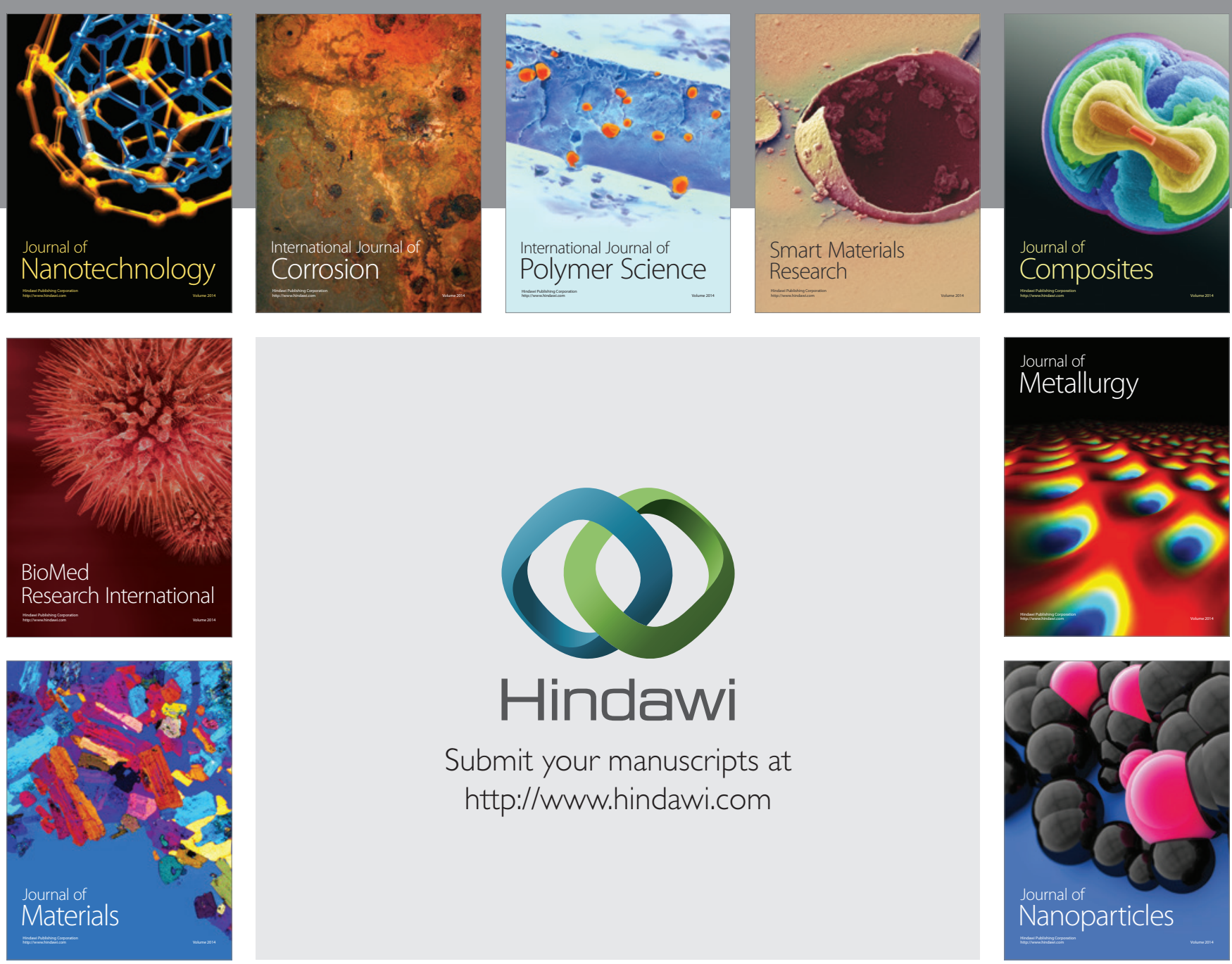

Submit your manuscripts at http://www.hindawi.com
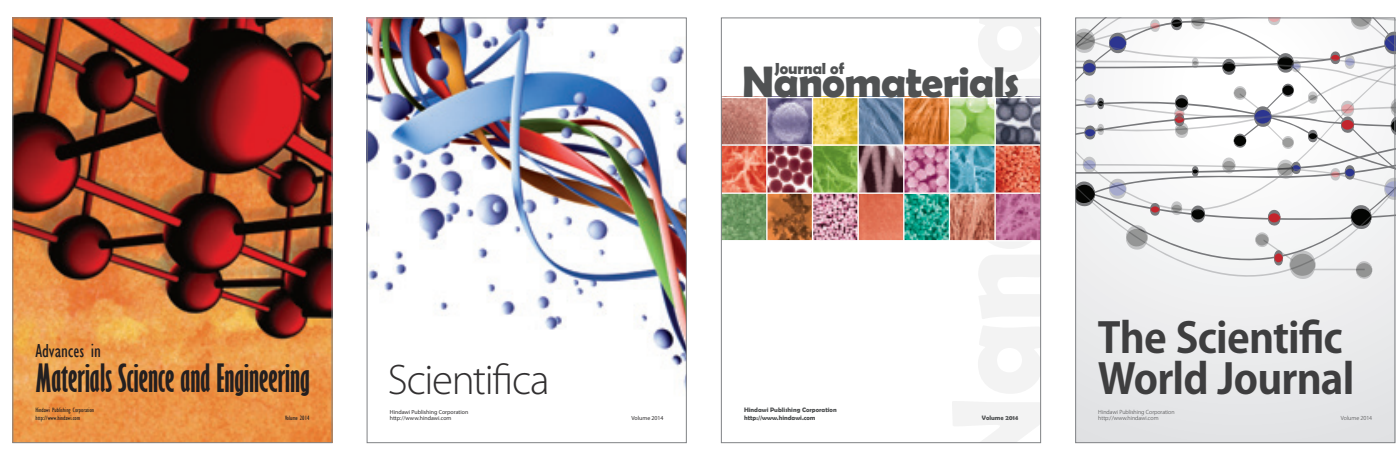

\section{The Scientific World Journal}
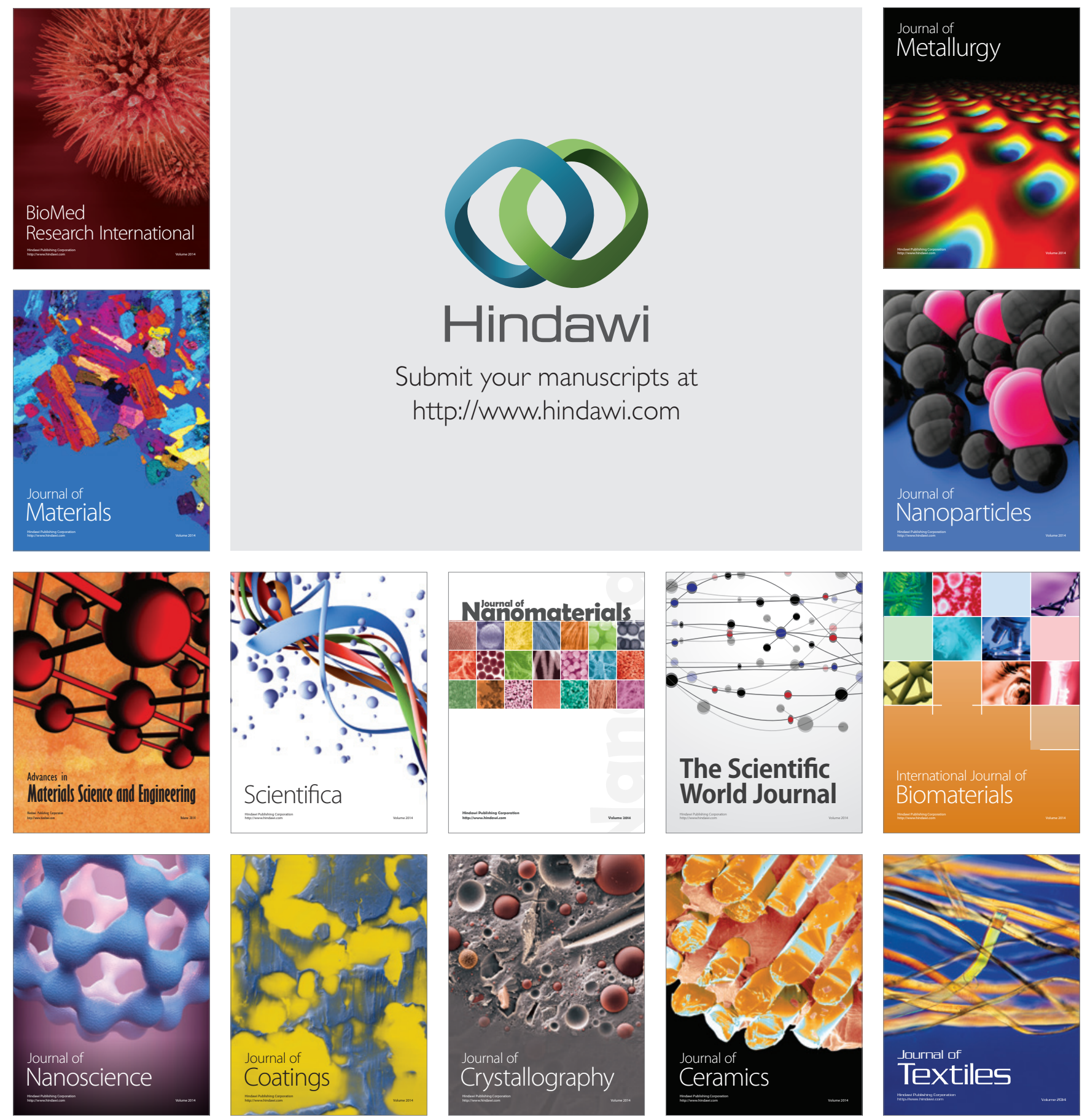